\title{
Frequency of double minute chromosomes and combined cytogenetic abnormalities and their characteristics
}

\author{
Yihui Fan • Renfang Mao $\cdot$ Hongpei Lv $\cdot$ Jie Xu • \\ Lei Yan $\cdot$ Yanhong Liu $\cdot$ Meng Shi $\cdot$ Guohua Ji • \\ Yang Yu $\cdot$ Jing Bai $\cdot$ Yan Jin $\cdot$ Songbin Fu
}

Received: 9 February 2010 /Revised: 7 July 2010 / Accepted: 18 July 2010 /Published online: 11 November 2010

(C) Institute of Plant Genetics, Polish Academy of Sciences, Poznan 2010

\begin{abstract}
Double minute chromosomes (DMs) are the cytogenetic hallmark of extra-chromosomal genomic amplification. The frequency of DMs in primary cancer and the cytogenetic features of DMs-positive primary cancer cases are largely unknown. To unravel these issues, we retrieved the Mitelman database and analyzed all DMs-positive primary cancerous karyotypes (787 karyotypes). The overall frequency of DMs is $1.4 \%$ (787 DMs-positive cases; total 54,398 cases). We found that DMs have the highest frequency in adrenal carcinoma (28.6\%, topography) and neuroblastoma (31.7\%, morphology). The frequencies of DMs in each tumor were much lower than in previous reports. The frequency of DMs in malignant cancers is significantly higher than in benign cancers, which confirms that DMs are malignant cytogenetic markers. DMs combined cytogenetic abnormalities are identified and sorted into two groups by principal component analysis (PCA), with one group containing $-4,-5,-8,-9$, $-10,-13,-14,-15,-16,-17,-18,-20,-21$, and -22 , and the other containing $-1 \mathrm{p},-5 \mathrm{q},+7$, and +20 . The prominent imbalance in DMs-positive cancer cases is chromosome loss. However, DMs-positive cancer cases, deriving from different
\end{abstract}

Y. Fan $\cdot$ R. Mao $\cdot$ H. Lv $\cdot$ J. Xu $\cdot$ L. Yan $\cdot$ Y. Liu $\cdot$ M. Shi $\cdot$ G. Ji $\cdot$

Y. Yu $\cdot$ J. Bai $\cdot$ Y. Jin $\cdot$ S. Fu $(\bowtie)$

Laboratory of Medical Genetics, Harbin Medical University,

No. 194, Xuefu Road,

Harbin 150081, China

e-mail: fusb@ems.hrbmu.edu.cn

S. Fu

e-mail: fusongbin@yahoo.com

S. Fu

Bio-pharmaceutical Key Laboratory of Heilongjiang Province,

No. 194, Xuefu Road,

Harbin 150081, China morphologic cancers, cannot be clearly divided into subgroups. Our large database analysis provides novel knowledge of DMs and their combined cytogenetic abnormalities in primary cancer.

Keywords Double minute chromosomes (DMs) . Karyotype $\cdot$ DMs-positive cancer cases $\cdot$ Imbalances

\section{Introduction}

Gene amplifications cause an increase in the gene copy number and, subsequently, elevate the expression of the amplified genes, which modify normal growth control and survival pathways. Double minute chromosomes (DMs) are the cytogenetic hallmark of extra-chromosomal genomic amplification (Hahn 1993). They are small, generally acentric, atelomeric, autonomously replicating chromatin bodies and are frequently detected in cytogenetic examinations of metaphase chromosomes in human tumor cells (Biedler et al. 1983). They have been found in a vast number of human neoplasias since the first description (Spriggs et al. 1962). Many oncogenes have been identified on DMs, for example, MYCN, C-MYC, EIF5A2, and MDM2 (Alitalo et al. 1983; Fakharzadeh et al. 1993; Guan et al. 2001; VanDevanter et al. 1990). Recently, some amplicon junctions were sequenced (Storlazzi et al. 2006). However, although DMs are appreciated at the single base pair level, the generation and function and even basic information such as the frequency of DMs in cancer remains largely unknown.

Benner et al. reported the presence of DMs in $93.5 \%$ of analytical tumors by reviewing 200 published tumors taken directly from patients (Benner et al. 1991). McGill et al. found DMs in 67 of the 76 examined ovarian cancers 


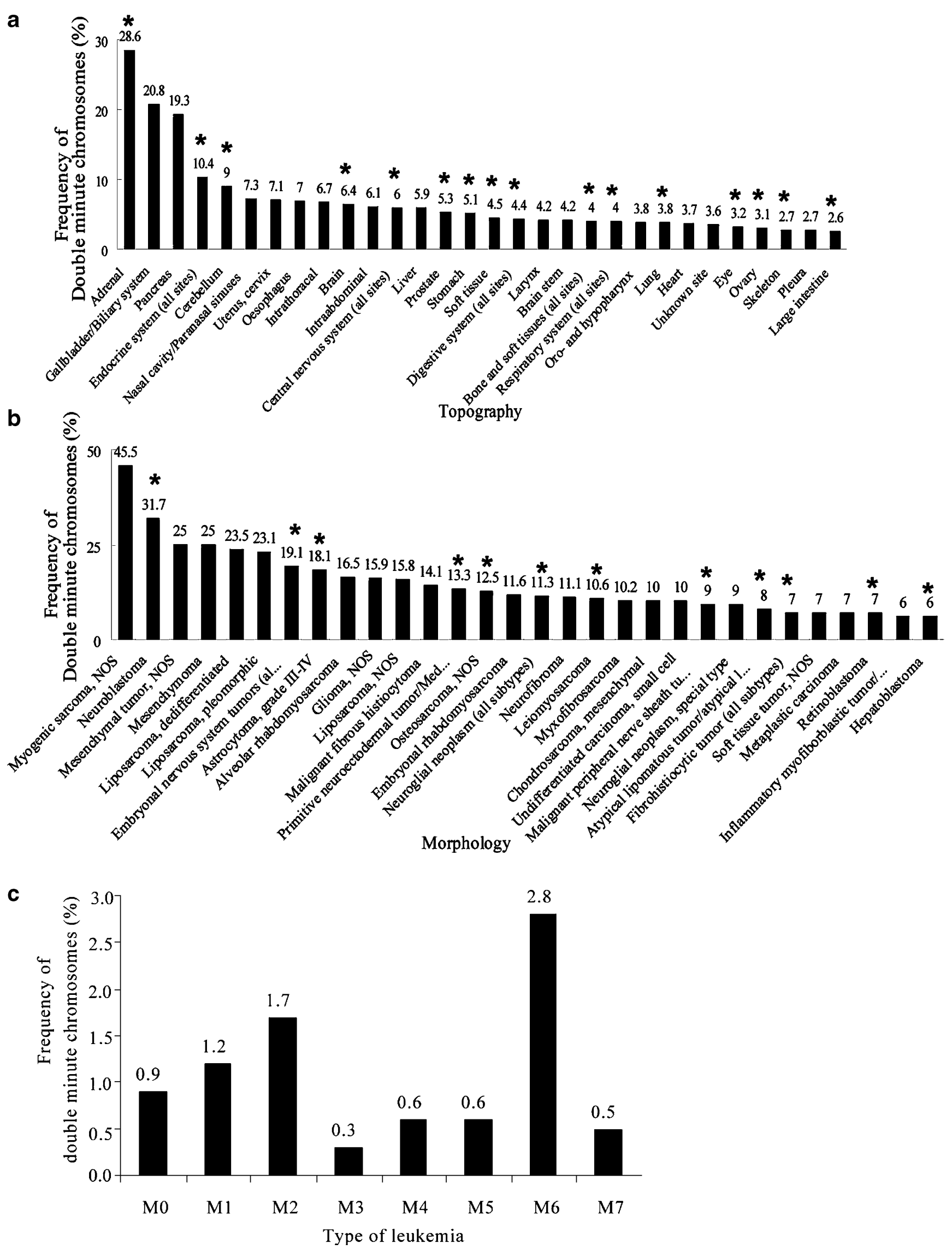


Fig. 1a-c Frequencies of double minute chromosomes (DMs) in tumors. a Frequencies of DMs in tumors classified by topography. Mitelman Cases Quick Searcher and Mitelman Cases Full Searcher were used as described on the website. In each topographic cancer, DMs-positive cancer cases were collected by the search item "*dmin*". *The number of analytic cases is more than 200. b Frequencies of DMs in tumors classified by morphology. *The number of analytic cases is more than 100 . c Frequencies of DMs in leukemia

(McGill et al. 1993). Gebhart et al. reported 50 DMspositive cases among 106 examined breast cancers (Gebhart et al. 1984). Gebhart also reviewed the available literature and found that the frequencies of DMs in ovarian cancer and breast cancer are $29 \%$ and $18 \%$, respectively (Gebhart 2005). The exact frequency of DMs in primary cancer is critically needed to be confirmed! Mitelman identified some common karyotypic features of different tumor types by statistically investigating the karyotypic database (Höglund et al. 2005). DMs are the cytogenetic hallmark of extra-chromosomal genomic amplification. However, the karyotypic features of DMs-positive primary cancer cases are not been characterized!

In this report, utilizing the largest chromosome aberrations database in cancer (Mitelman Database of Chromosome Aberrations and Gene Fusions in Cancer, http://cgap.nci.nih. gov/Chromosomes/Mitelman), we find that the frequencies of DMs in each tumor are much lower than in previous reports. DMs have the highest frequency in adrenal carcinoma $(28.6 \%$, topography) and neuroblastoma $(31.7 \%$, morphology). We further analyze all DMs-positive karyotypes (787 DMs- positive cases) and DMs combined cytogenetic abnormalities and present their characteristics.

\section{Materials and methods}

Data retrieval and conversion

The Mitelman Database of Chromosome Aberrations and Gene Fusions in Cancer (http://cgap.nci.nih.gov/Chromosomes/ Mitelman; updated October 2007) provides a powerful tool for statistical analyses of the chromosome aberrations in cancer (Shimizu et al. 2007). We collected all DMs-positive or homogeneously staining region (HSR)-positive cancer cases by the following strategy: (1) abnormality "* dmin*" for DMspositive cases; (2) abnormality "*hsr*" for HSR-positive cases. Mitelman Cases Quick Searcher and Mitelman Cases Full Searcher were used as described on the website.

Karyotypes from 778 DMs-positive cases were downloaded as text files. The International System for Human Cytogenetic Nomenclature (ISCN) karyotypes were converted into binary data denoting the status of each chromosome band as 1 if gained, -1 if lost, and 0 if neither gained nor lost. When constructing the imbalance map, unknown band data such as $+\operatorname{der}(?) t(? ; 1 ; 7)$ were excluded and only the clone containing DMs for each case was entered. If the aberrations were restricted in the arm of the chromosome, they were designated as the corresponding chromosome arm aberrations. Chromosomes and chromosome arms gained or lost in $>10 \%$ of the tumors were selected for further analysis.
Fig. 2a-c DMs are significantly associated with the tumor malignancy. Frequencies of DMs in three pair cancers. a Astrocytoma grade I-II to astrocytoma grade III-IV. b Benign epithelial neoplasms to malignant epithelial neoplasms. c Benign epithelial tumor special type to malignant epithelial tumor special type
$\mathbf{A}$

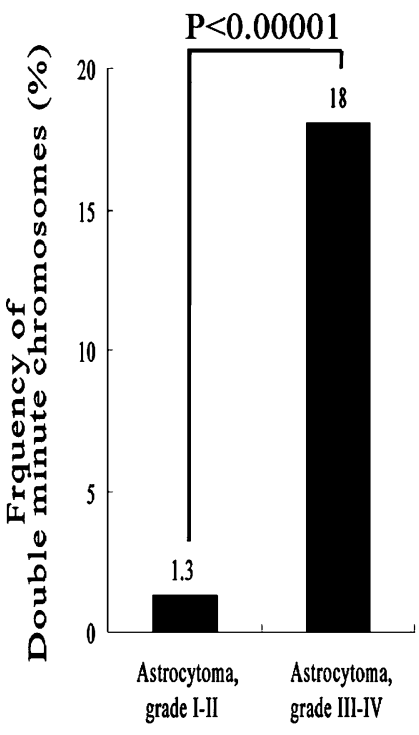

B

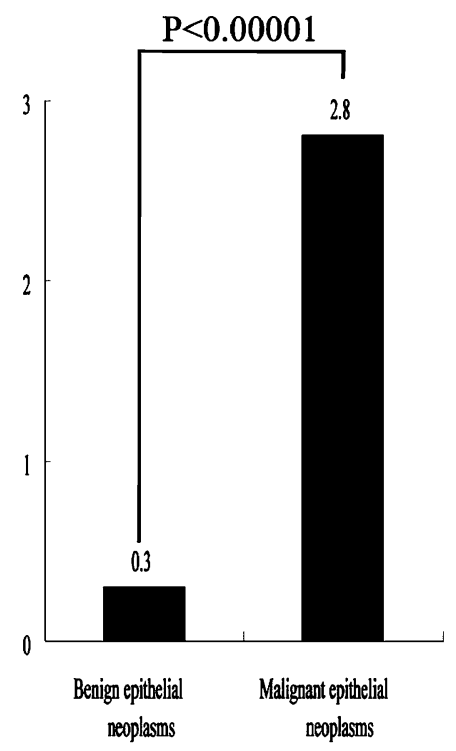

C

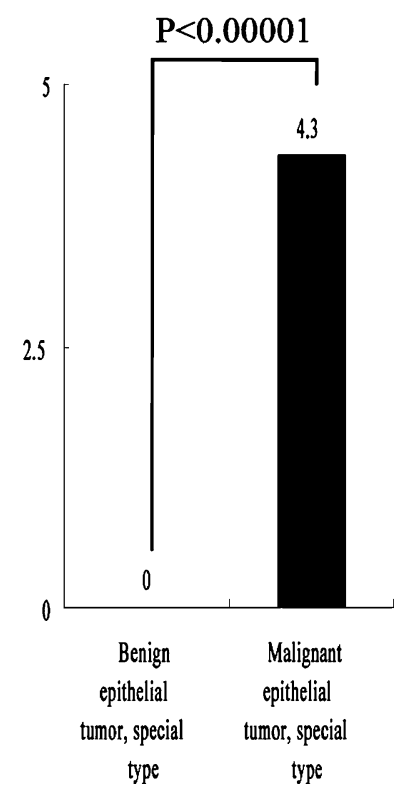



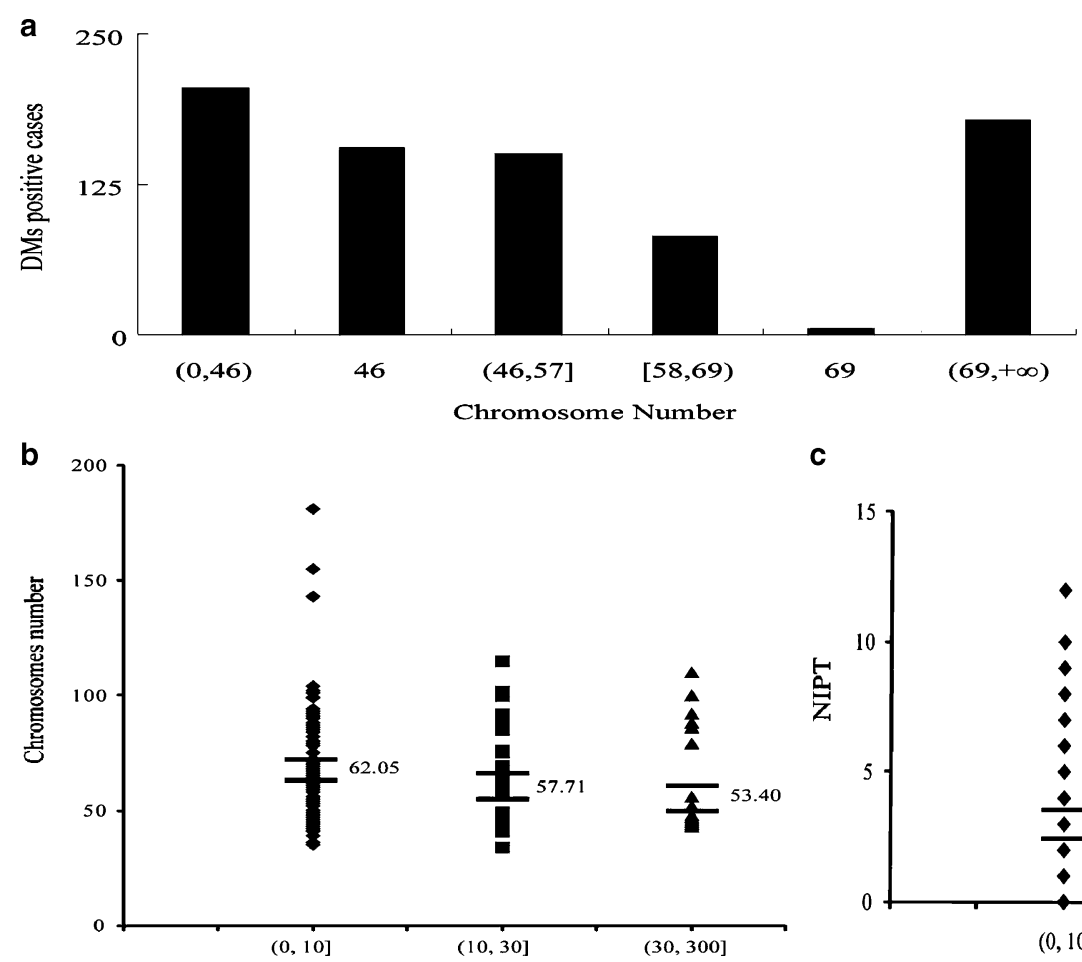

c

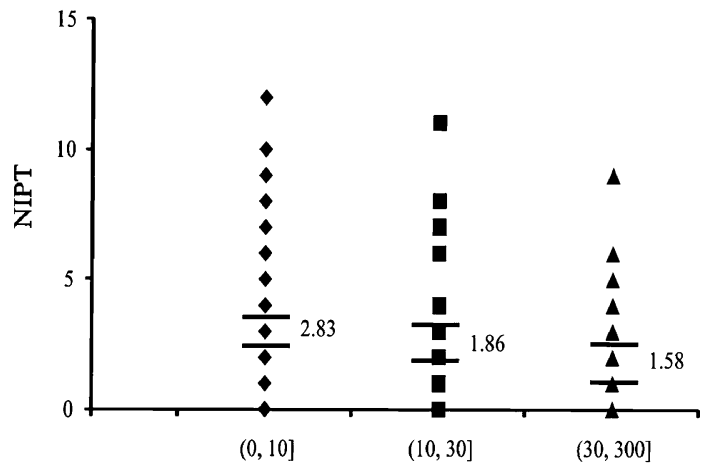

Fig. 3a-c Distribution of chromosome number in DMs-positive cancers. a Number of DMs-positive cancer cases in six subgroups according to the number of chromosomes. Hypodiploid includes the most DMs-positive cases (27.2\%). b Distributions of chromosome

\section{Statistical analysis}

Principal component analysis (PCA) was performed using the SPSS 10.0 program (SPSS inc., Chicago, IL, USA). Principal components are linear combinations of the original variables, orthogonal, and ordered with respect to their variance so that the first principal component has the largest variance. Imbalances were used as variables and the individual tumors as the observations. For clustering cases, these cases were used as variables and the imbalances as observations. The Chi-squared test was performed using the SPSS program and statistical significance was accepted at the $p<0.05$ level.

\section{Results}

Summary of the DMs-positive cancer cases

As of February 2008, there are a total of 54,398 cases in the Mitelman database. Among them, 787 (1.4\%) cases are DMs-positive and $467(0.9 \%)$ cases are HSR-positive. The number of dual-positive cases is $44(0.08 \%)$. In DMspositive cases, the ratio of males to females is 1.08 (401/ 373). Karyotypes from 778 DMs-positive cases (to date on October 2007) were downloaded as text files for further analysis. Twenty-four (3.1\%) cases present DMs as the sole number in three subgroups according to the number of DMs. c Distributions of the number of imbalances per tumor (NIPT) in three subgroups according to the number of DMs

abnormality. Two hundred and thirty-five (30.2\%) cases counted the number of DMs and 775 (99.6\%) cases provided the number of chromosomes.

Frequencies of DMs in cancer

Frequencies of DMs in cancer according to topography are shown in Fig. 1a. The frequency in adrenal carcinoma is the highest $(28.6 \%)$. Frequencies in the endocrine system, cerebellum, brain, central nervous system (all sites), prostate, stomach, soft tissue, digestive system (all sites), bone and soft tissues (all sites), respiratory system (all sites), lung, eye, ovary, skeleton, and large intestine carcinoma are $10.4 \%, 9 \%$, $6.4 \%, 6 \%, 5.3 \%, 5.1 \%, 4.5 \%, 4.4 \%, 4 \%, 4 \%, 3.8 \%, 3.2 \%$, $3.1 \%, 2.7 \%$, and $2.6 \%$, respectively. The number of analyzed cases in the above tumors is more than 200, thus, the frequencies are more credible. Figure $1 \mathrm{~b}$ presents the frequencies of DMs in cancer according to morphology. In the tumors, which include more than 100 cases, neuroblastoma has the highest frequency of DMs (31.7\%). The frequencies in embryonic nervous system tumors (all subtypes), astrocytoma (grade III-IV), primitive neuroectodermal tumor, osteosarcoma (NOS), neuroglial neoplasm (all subtypes), leiomyosarcoma, malignant peripheral nerve sheath tumor, atypical lipomatous tumor, fibrohistiocytic tumor (all subtypes), retinoblastoma, and hepatoblastoma are 19.1\%, 
a

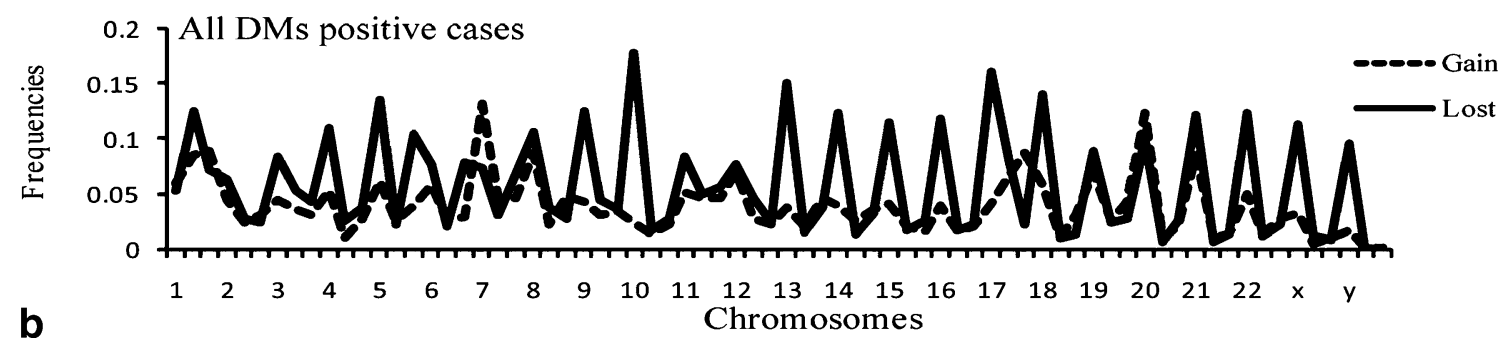

b

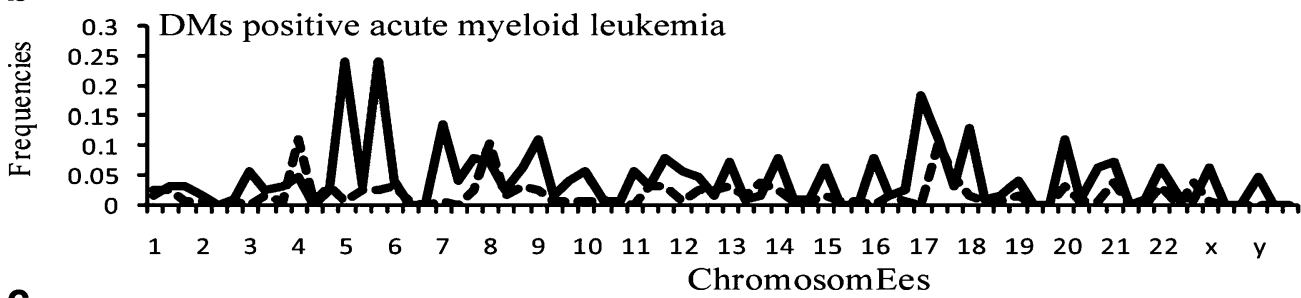

C
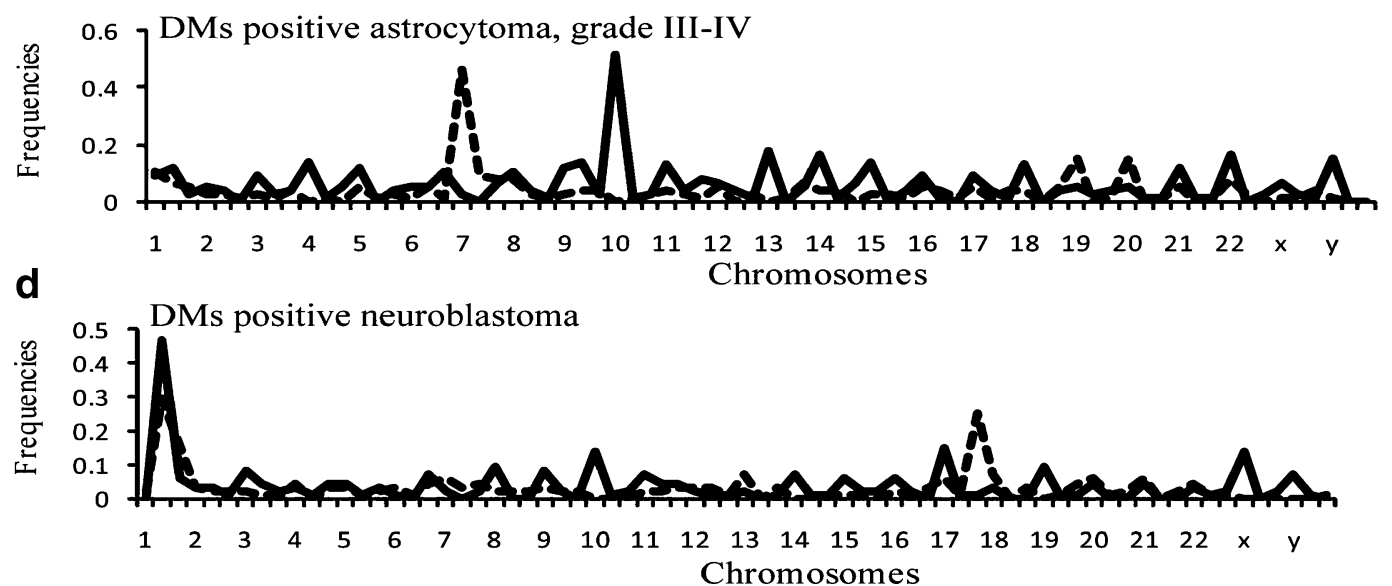

Fig. 4a-d Karyotypic profiles of DMs-positive cancers. a All cases. b Acute myeloid leukemia. c Astrocytoma grade III-IV. d Neuroblastoma cancers

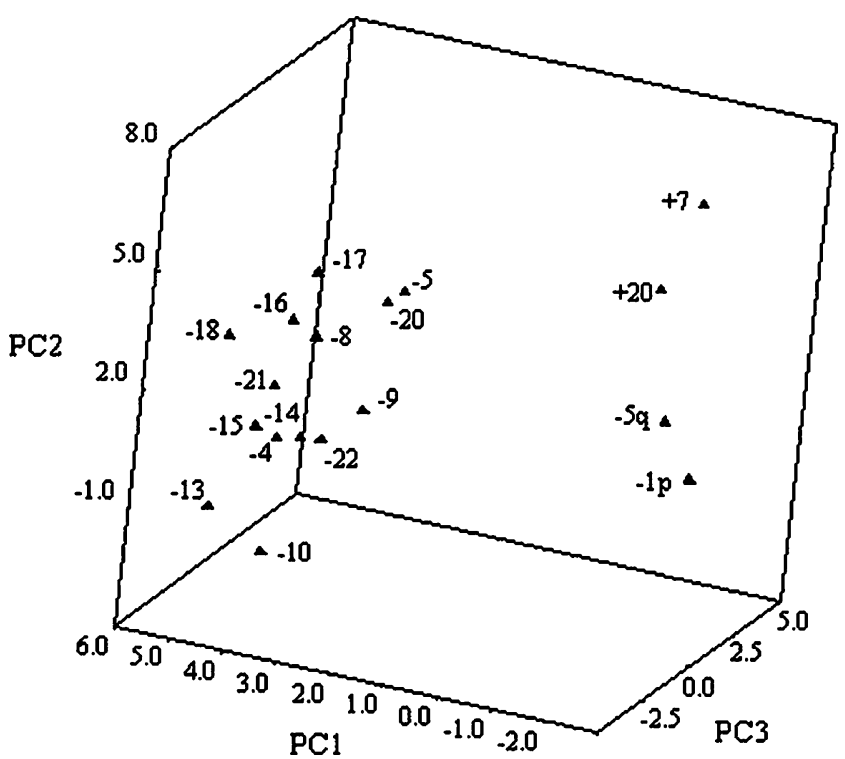

Fig. 5 Three-dimensional presentation of recurrent imbalances after principle component analysis (PCA)
$18.1 \%, 13.3 \%, 12.5 \%, 11.3 \%, 10.6 \%, 9.3 \%, 7.7 \%, 7.1 \%$, $6.6 \%$, and $6.0 \%$, respectively. The frequency of DMs in M0 to $\mathrm{M} 7$ of leukemia ranges from $0.3 \%$ to $2.8 \%$ (Fig. 1c).

Three pair cancers provided the pathological stage in the Mitelman database (astrocytoma grade I-II to astrocytoma grade III-IV; benign epithelial neoplasm to malignant epithelial neoplasm; and benign epithelial tumor special type to malignant epithelial tumor special type). To examine the association between DMs and tumor malignancy, a Chi-square test was carried out. As shown in Fig. 2, DMs are statistically significantly associated with the tumor malignancy $(p<0.001, n=7,576)$.

Karyotypic profile of DMs-positive cancer

DMs-positive cases are subdivided into six groups according to the number of chromosomes $(<46 ; 46 ; 46<\leq 57 ; 58 \leq<69$; $69 ; 69<$ ). Figure $3 \mathrm{a}$ presents the number of cases in each group. Most of the DMs-positive cases were hypodiploid $(205,27.2 \%)$. Two hundred and thirty-five cases counted the 

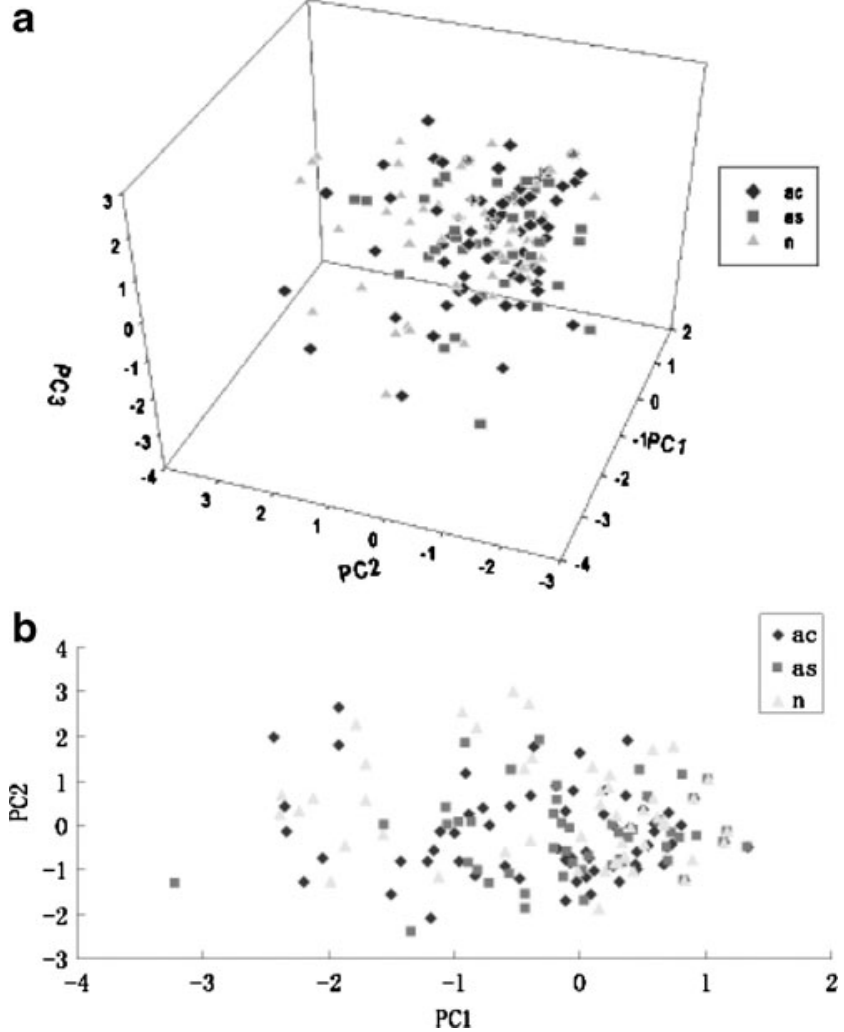

Fig. 6a, b Three- and two-dimensional presentations of three DMspositive morphologic cancer cases after PCA. a Three-dimensional presentation of three DMs-positive morphologic cancer cases. b Twodimensional presentation of three DMs-positive morphologic cancer cases. $a c$ acute myeloid leukemia; as astrocytoma grade III-IV; $n$ neuroblastoma

number of DMs. These cases were sorted into three groups based on the number of DMs $(\leq 10 ; 10<\leq 30 ; 30 \leq)$. Distributions of the number of chromosomes in each group are shown in Fig. 3b. The chromosome number was found to decrease with the accumulation of DMs.

The karyotypic profiles of all DMs-positive cancer cases are presented in Fig. 4a. In all DMs-positive cancer cases, the most frequently observed imbalances were -10 (17.9\%), -17 (16.1\%), -13 (15.2\%), -18 (14.1\%), -5 $(13.6 \%)$, and $+7(13.1 \%)$. The prominent imbalance in DMs-positive cancer is chromosome loss. Three hundred and fifty-three $(45.4 \%)$ cases present with translocation, and the frequency of marker chromosomes is $36.8 \%$ (286/ 778). To see if DMs-positive cancers have common features or vary with morphology, we constructed karyotypic profiles of DMs-positive acute myeloid leukemia, astrocytoma grade III-IV, and neuroblastoma cancers. As shown in Fig. 4 , the most frequently observed imbalances were -5 $(24.0 \%),-5 q(24.0 \%)$, and $-17(18.4 \%)$ in DMs-positive acute myeloid leukemia, $+7(46.2 \%)$ and $-10(51.3 \%)$ in DMs-positive astrocytoma grade III-IV, and $-1 \mathrm{p}(47.1 \%)$ in DMs-positive neuroblastoma.
Principal component analysis (PCA) of imbalances and clusters

Imbalances recorded in $>10 \%$ of the carcinomas were defined as the recurrence for further PCA. Aberrations of the $\mathrm{X}$ or $\mathrm{Y}$ chromosome were excluded from the analyses, because DMs-positive cases are almost equal among males and females. A total of 18 recurrent imbalances were identified, including $-1 \mathrm{p},-4,-5,-5 \mathrm{q},+7,-8,-9,-10$, $-13,-14,-15,-16,-17,-18,+20,-20,-21$, and -22 . Each case was then assessed for the presence or absence of recurrent imbalances. Cases with at least one of the chosen imbalances were selected for further analysis, resulting in 506 cases. The number of imbalances per tumor (NIPT) was counted. Distributions of the NIPT in each group sorted by the number of DMs are shown in Fig. 3c. The NIPT decreased with the accumulation of DMs. We performed PCA of the karyotypic data, which revealed two distinct clusters of aberrations, one containing $-4,-5$, $-8,-9,-10,-13,-14,-15,-16,-17,-18,-20,-21$, and -22 , and the other containing $-1 \mathrm{p},-5 \mathrm{q},+7$, and +20 (Fig. 5). To group the DMs-positive cases, PCA was performed using tumor cases as variables and the imbalances as cases. DMs-positive cancer cases cannot be clearly divided into subgroups (data not shown), but for a DMspositive morphologic cancer, cases were more frequently located in the same region. A scatter plot of three different morphologic cancers indicate the same results (Fig. 6).

\section{Discussion}

We retrieved the overall cases from the Mitelman database and 778 DMs-positive cases were downloaded as text files for further analysis. We found that frequencies of DMs in each kind of cancer are much lower than in previous reports. DMs have the highest frequency in adrenal carcinoma (28.6\%, topography) and neuroblastoma (31.7\%, morphology). The frequencies of DMs in M0 to M7 range from $0.3 \%$ to $2.8 \%$ (Fig. 1c), much lower than that previously reported (3\% to 11\%) (Keung et al. 1997; Kuttler and Mai 2007). In the breast, ovarian, and lung cancers, the frequencies of DMs are $0.8 \%, 3.1 \%$, and $3.8 \%$, respectively, which is also much lower than that of previous reports (breast: 18\%; ovarian: 29\%; small-cell lung cancer (SCLC): 20\%-30\%; non-SCLC (NSCLC): 10\%-20\%) (Gebhart 2005). Three paired cancers provided the pathological stage in the Mitelman database and yielded powerful evidence that DMs can reliably reflect the advanced stage of malignancy (Fig. 2), suggesting that it can be taken as a malignant cytogenetic marker. The frequency of DMs significantly increases with the progression of cancer. A ten-fold increase was seen in malignant 
epithelial neoplasms compared to benign epithelial neoplasms. Thus, we infer that the above frequency inconsistency is due to the different pathological stages of cases investigated in each report. Our data is obtained from a large Database of Chromosome Aberrations and Gene Fusions in Cancer. Therefore, the frequencies of DMs in each cancer are objective. However, the frequencies presented here are gross, without regard to the pathological stages. Therefore, in some investigative cohorts, especially for malignant cancer cases, the frequencies of DMs may be ten to 20 times higher than the frequencies presented in our data.

The DMs combined cytogenetic abnormalities comprised two distinct clusters, one containing $-4,-5,-8,-9,-10,-13$, $-14,-15,-16,-17,-18,-20,-21$, and -22 , and the other containing $-1 p,-5 q,+7$, and +20 . The prominent imbalance in DMs-positive cancer is chromosome loss. DMs-positive cancer cases were divided into six groups according to the number of chromosomes. The hypodiploid group included the most DMspositive cases (27.2\%). Morales et al. reported that passive loss of the DHFR amplicon by the withdrawal of MTX results in MTX-sensitive cells exhibiting a substantial reduction of their capacity or even an incapacity to generate resistance when submitted to a second cycle of MTX treatment (Morales et al. 2009). This phenomenon may be due to chromosome loss. To adapt cells to stress conditions, target DNA fragments are released from the original chromosomes and amplify as DMs. After withdrawal of the drug, DMs will be lost and leave impaired chromosomes. The cells with impaired chromosomes have an incapacity to generate resistance when they are submitted to the same repeated stress conditions. Based on this inference, we postulate that waiting for the complete loss of DMs is essential before starting the second round of treatment in DMs-positive cancer cases.

In summary, by retrieving the Mitelman database, we provided novel knowledge of DMs and their combined cytogenetic abnormalities. Our findings will be beneficial for further study of the generation and function of DMs.

Acknowledgments This research was supported by the National Natural Science Foundation (nos. 30771198, 30801350, and 30800268) and the Heilongjiang Province Office of Science and Technology Education research project (nos. 11531446 and 10553036).

\section{References}

Alitalo K, Schwab M, Lin CC, Varmus HE, Bishop JM (1983) Homogeneously staining chromosomal regions contain amplified copies of an abundantly expressed cellular oncogene (c-myc) in malignant neuroendocrine cells from a human colon carcinoma. Proc Natl Acad Sci USA 80:1707-1711

Benner SE, Wahl GM, Von Hoff DD (1991) Double minute chromosomes and homogeneously staining regions in tumors taken directly from patients versus in human tumor cell lines. Anticancer Drugs 2:11-25

Biedler JL, Chang TD, Meyers MB, Peterson RH, Spengler BA (1983) Drug resistance in Chinese hamster lung and mouse tumor cells. Cancer Treat Rep 67:859-867

Fakharzadeh SS, Rosenblum-Vos L, Murphy M, Hoffman EK, George DL (1993) Structure and organization of amplified DNA on double minutes containing the $\mathrm{mdm} 2$ oncogene. Genomics $15: 283-290$

Gebhart E (2005) Double minutes, cytogenetic equivalents of gene amplification, in human neoplasia-a review. Clin Transl Oncol 7:477-485

Gebhart E, Brüderlein S, Tulusan AH, von Maillot K, Birkmann J (1984) Incidence of double minutes, cytogenetic equivalents of gene amplification, in human carcinoma cells. Int $\mathrm{J}$ Cancer 34:369-373

Guan XY, Sham JS, Tang TC, Fang Y, Huo KK, Yang JM (2001) Isolation of a novel candidate oncogene within a frequently amplified region at 3q26 in ovarian cancer. Cancer Res 61:3806-3809

Hahn PJ (1993) Molecular biology of double-minute chromosomes. Bioessays 15:477-484

Höglund M, Frigyesi A, Säll T, Gisselsson D, Mitelman F (2005) Statistical behavior of complex cancer karyotypes. Genes Chromosomes Cancer 42:327-341

Keung YK, Cobos E, Morgan D, Whitehead RP, Tonk V (1997) Double minute chromosomes and myelodysplastic syndrome: a case report and literature review. Cancer Genet Cytogenet 97:94-96

Kuttler F, Mai S (2007) Formation of non-random extrachromosomal elements during development, differentiation and oncogenesis. Semin Cancer Biol 17:56-64

McGill JR, Beitzel BF, Nielsen JL, Walsh JT, Drabek SM, Meador RJ, Von Hoff DD (1993) Double minutes are frequently found in ovarian carcinomas. Cancer Genet Cytogenet 71:125-131

Morales C, García MJ, Ribas M, Miró R, Muñoz M, Caldas C, Peinado MA (2009) Dihydrofolate reductase amplification and sensitization to methotrexate of methotrexate-resistant colon cancer cells. Mol Cancer Ther 8:424-432

Shimizu N, Misaka N, Utani K (2007) Nonselective DNA damage induced by a replication inhibitor results in the selective elimination of extrachromosomal double minutes from human cancer cells. Genes Chromosomes Cancer 46:865-874

Spriggs AI, Boddington MM, Clarke CM (1962) Chromosomes of human cancer cells. Br Med J 2:1431-1435

Storlazzi CT, Fioretos T, Surace C, Lonoce A, Mastrorilli A, Strömbeck B, D'Addabbo P, Iacovelli F, Minervini C, Aventin A, Dastugue N, Fonatsch C, Hagemeijer A, Jotterand M, Mühlematter D, Lafage-Pochitaloff M, Nguyen-Khac F, Schoch C, Slovak ML, Smith A, Solè F, Van Roy N, Johansson B, Rocchi M (2006) MYC-containing double minutes in hematologic malignancies: evidence in favor of the episome model and exclusion of MYC as the target gene. Hum Mol Genet 15:933942

VanDevanter DR, Piaskowski VD, Casper JT, Douglass EC, Von Hoff DD (1990) Ability of circular extrachromosomal DNA molecules to carry amplified MYCN proto-oncogenes in human neuroblastomas in vivo. J Natl Cancer Inst 82:1815-1821 